UDC 519.63; 519.684

\author{
*Issakhov A., Shubay A. \\ Faculty of Mechanics and Mathematics, \\ al-Farabi Kazakh National University, Almaty, Kazakhstan \\ *e-mail: alibek.issakhov@gmail.com
}

\title{
Mathematical modelling of blood flow in vascular system of the brain with pathological changes
}

\begin{abstract}
The paper presents numerical study of blood flow in vascular system without stenosis and with pathological changes. The Navier - Stokes system of equation is used for modeling of liquid flow in blood vessels. The numerical algorithm was based on projection method. At first stage, it is assumed that the transfer of the movement of amount occurred is due to convection and diffusion. The intermediate regions of velocity are determined by using the 5-step Runge - Kutta method algorithm. At second stage the intermediate velocity field is used to define the pressure field. The Poisson equation for pressure field is solved by Jacobi method. The numerical results are checked by comparing analytical solutions for the Poiseuille flow with numerical results. The influence of the blood velocity flow on behavior of the liquid in vascular system of brain was investigated. One of the characteristic features of blood flow in blood vessels affected by stenosis is an appearance of vortex currents, which manifests as a pathology, as opposite to normal blood circulation. The results of this work can be used in mathematical modeling investigations of blood circulation in brain and solving problems in practical medicine.
\end{abstract}

Key words: Navier-Stokes equation, Poiseuille flow, atherosclerotic plaque, stenosis, pathological changes in the blood vessels.

One of the most difficult and important problems of medicine is curing of the pathology of cerebral circulation. Because of the cerebrovascular disease spreading prevention and operative therapy of dysfunction of brain circulation very actual nowadays. The death rate from insult reaches 35\% in Kazakhstan (450000 insults), herewith the risk of insult increases with age rise, particularly at the age of $65-80$. Insult is serious complication of vascular heart diseases, which is located in the first three reasons of death and in some cases the death rate of insult exceeds the death rate of heart attack. The probability of disability is very high in case of patients suffered from insult, $20 \%$ of them return to work, $60 \%$ of patients have neurological disorders, $20 \%$ of patients need constant nursing care. The rise of the patients number with vascular brain diseases is depend on a lot of factors and connected with the age rise and the increase of weight of elderly people.

\section{Formulation of the task}

In the work is made formulation of the problem and the equations of blood flow in vessels of the thick - wall hyper elasticity vessel. It is assumed that blood is homogeneous incompressible Newtonian medium. Vessel is considered as thick wall cylinder form hyper elastic material. Blood is affected by changing pressure gradient.

For a description of blood movement, the no stationary two - dimensional Navier - Stokes equations with constant density and kinematic viscosity are used $[1,2]$ :

$$
\left\{\begin{array}{l}
\frac{\partial u}{\partial x}+\frac{\partial v}{\partial y}=0 \\
\frac{\partial u}{\partial t}+u \frac{\partial u}{\partial x}+v \frac{\partial u}{\partial y}=-\frac{1}{\rho} \frac{\partial p}{\partial x}+\vartheta\left(\frac{\partial^{2} u}{\partial x^{2}}+\frac{\partial^{2} u}{\partial y^{2}}\right) \\
\frac{\partial v}{\partial t}+u \frac{\partial v}{\partial x}+v \frac{\partial v}{\partial y}=-\frac{1}{\rho} \frac{\partial p}{\partial y}+\vartheta\left(\frac{\partial^{2} v}{\partial x^{2}}+\frac{\partial^{2} v}{\partial y^{2}}\right)
\end{array}\right.
$$

where $p$ - pressure, $u, v$ - velocity component, $\rho$ density, $x, y$ - space coordinates, $t$ - time, $v$ kinematic viscosity. In this work by using this system of equation is modeled two problems stenos is in cerebral vessels are modeled in the work.

First problem is healthy vessel (figure 1).

Printed in Kazakhstan 


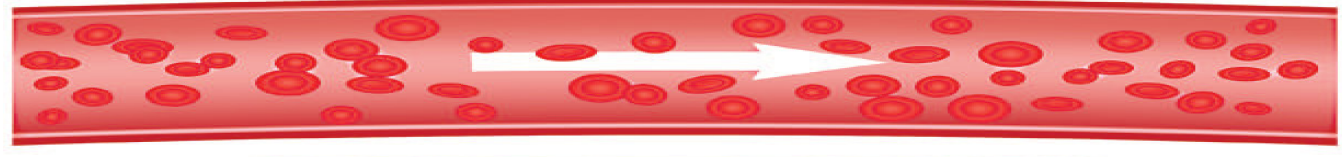

Figure 1 - Healthy vessel

Second problem is stenosis on one of the vascular wall (figure 2):

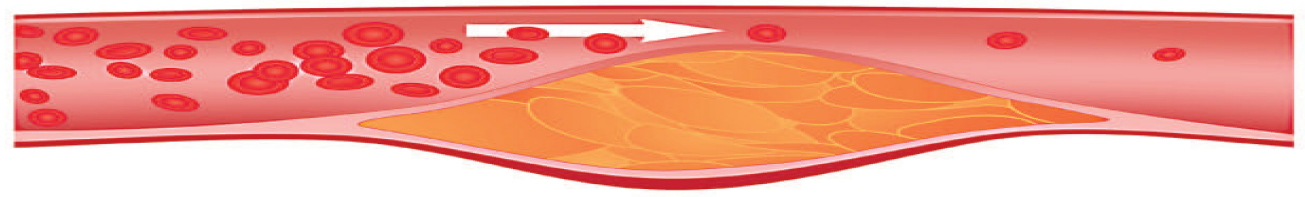

Figure2 - Vessel with atheroma

\section{Numerical algorithm}

For solving of the system of equations (1) is used projection method [7, 10]. At first stage, it is assumed that the transfer of the movement of amount occurred is due to convection and diffusion. The intermediate regions of velocity are determined by using the 5-step Runge - Kutta method algorithm. At second stage the intermediate velocity field is used to define the pressure field. The Poisson equation for pressure field is solved by Jacobi method. At the third stage, it is assumed that the transfer if carried out only by pressure gradient. Below is given the numerical algorithm in mathematical form $[8,9]$ :

I. $\int_{\Omega} \frac{\vec{u}^{*}-\vec{u}^{n}}{\tau} d \Omega=-\oint_{\partial \Omega}\left(\nabla \vec{u}^{n} \vec{u}^{*}-v \Delta \vec{u}^{*}\right) n_{i} d \Gamma$

II. $\oint_{\partial \Omega}(\Delta p) d \Gamma=\int_{\Omega} \frac{\overrightarrow{\nabla u}^{*}}{\tau} d \Omega$
III. $\frac{\vec{u}^{n+1}-\vec{u}^{*}}{\tau}=-\nabla p$

\section{The results of numerical estimations}

The system of equations (1)is closed by following boundary conditions: for the velocity components on the wall no slip is set, in inlet parabolic profile, in outlet- outlet boundary conditions. For the pressure the Neumann conditions were set in all boundaries.

Poiseuille flow (the first problem) is viscous flow going through a tube with determined diameter and theoretically infinite length. At figure 3, a geometric parameters are given, which were used in solving of this problem. The basis of the research work is taken from work Schlichting G. [6].

In work [6] the tube with length $L$ and radius 1 were used with Reynolds number $R e=100$. And $\frac{\mathrm{L}}{1}=10$ (figure 3 )

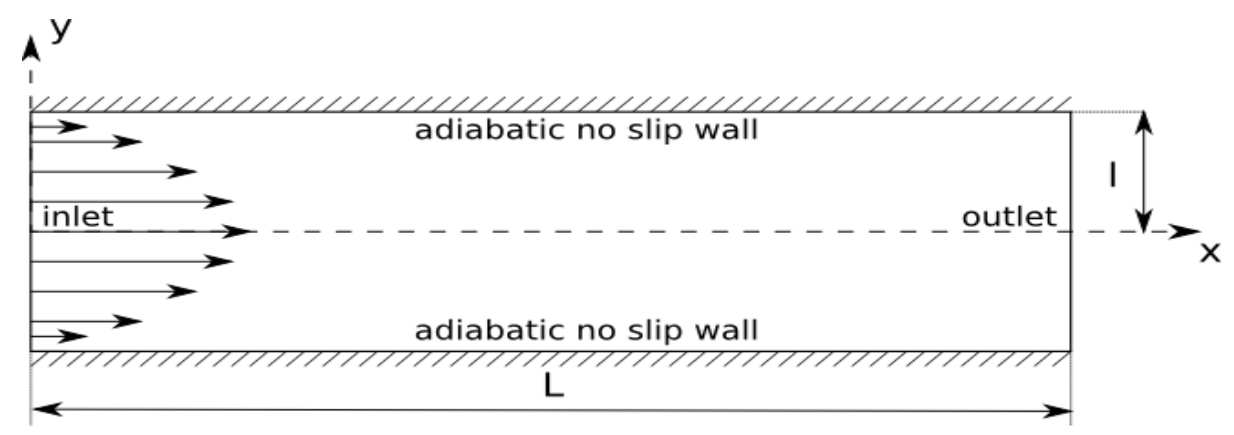

Figure 3 - Computational domain 
At figure 4, shown comparison of numerical and analytical solution of streamwise velocity at points

$x / L=0$ and $x / L=9$ is presented. Results taken form research matches with analytical solution very well.

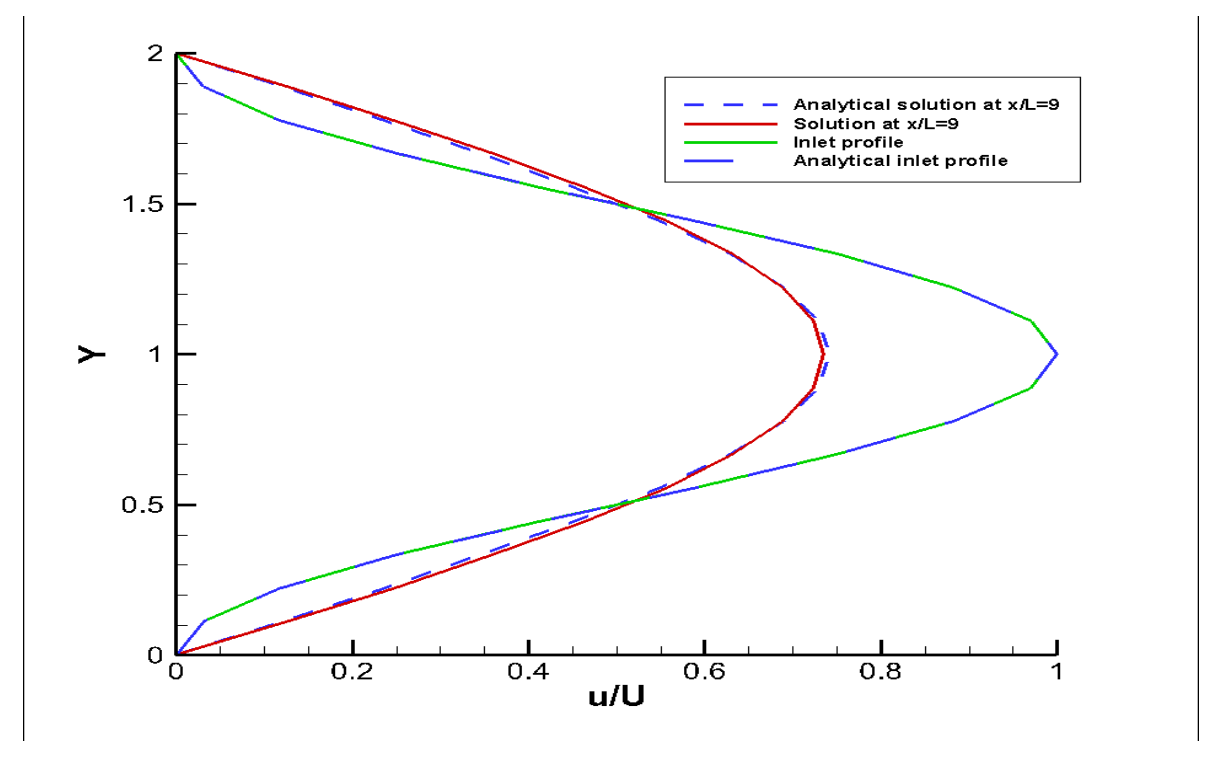

Figure 4-Velocity profile on points $x / L=0$ and $x / L=9$

At figure 5, the velocity field for the number $\mathrm{Re}=100$ is presented for different time lays. From figure 5, we can make following conclusions, that the speed of blood flow in the norm is laminar and the velocity profile is parabolic. The movement of blood speed in the center of vessel turns out much bigger than movement velocity near the vascular wall. Wherein the blood layer adjoining to the vascular vessel is almost stationary, the next blood layer moves a small distance, the blood portion located in the center part of vascular vessel moves big distance. Thus, the velocity profile distributionforms in vessels.

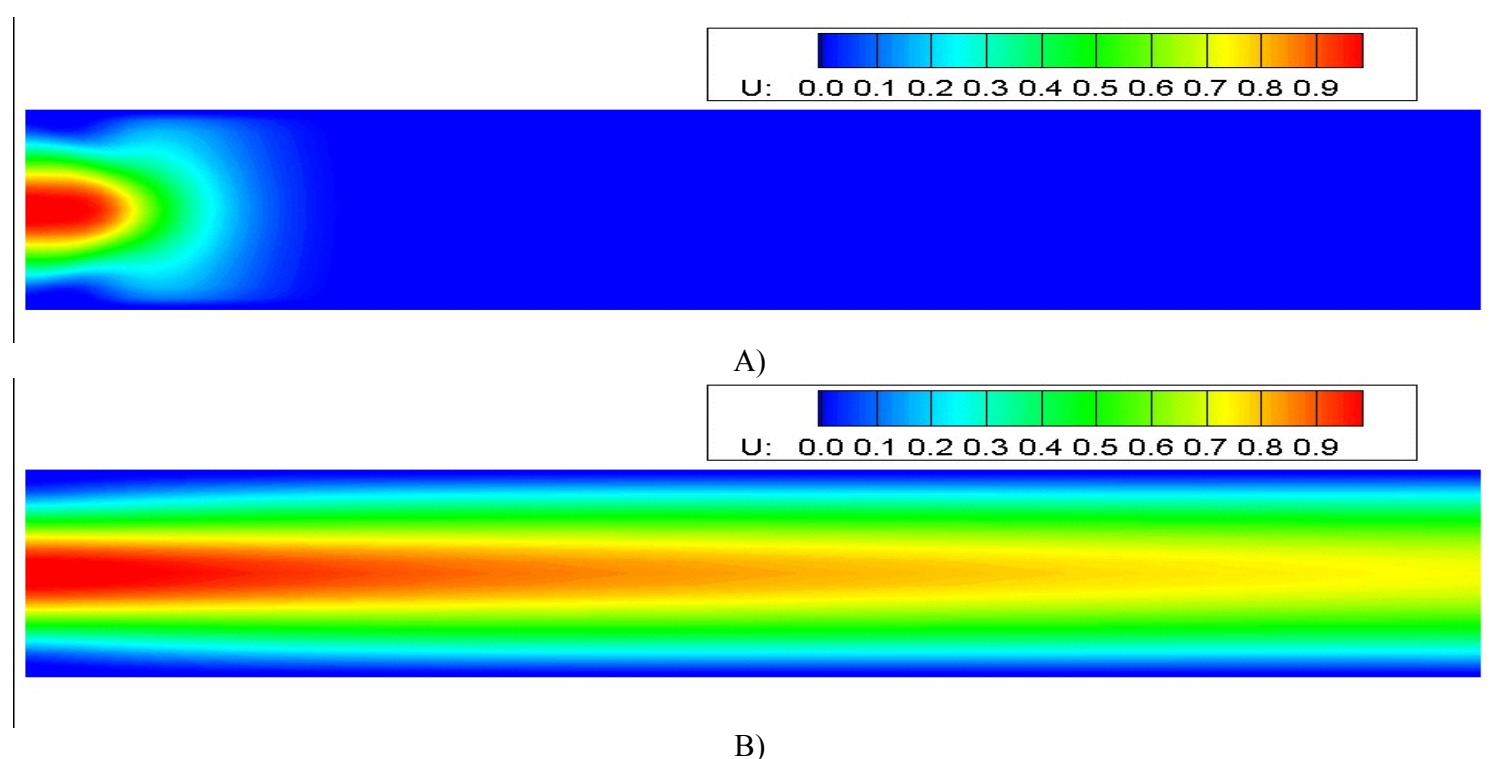

Figure 5 - Contour of velocity fieldRe $=100$ at various time layers $(A) t=0, B$ ) $t=10$ ) 
Table 1 - Properties of vessels and blood.

\begin{tabular}{|c|c|c|c|c|c|}
\hline Vessels & $D[\mathrm{~cm}]$ & $V\left[\frac{\mathrm{cm}}{\mathrm{s}}\right]$ & $\rho\left[\frac{\mathrm{gr}}{\mathrm{cm}}\right]$ & $\mu\left[\frac{\mathrm{gr}}{\mathrm{cm} \cdot \mathrm{s}}\right]$ & $\mathrm{T}\left[{ }^{\circ} \mathrm{C}\right]$ \\
\hline $\begin{array}{c}\text { Artery } \\
\text { 1) Carotid }\end{array}$ & $0,15-0,25$ & 20 & 1,05 & $0,0255-0,03$ & $40-41$ \\
\hline 2)Vertebral artery & $0,15-0,25$ & 25 & 1,05 & $0,0255-0,03$ & $40-41$ \\
\hline 3)Infraorbital artery & $0,15-0,25$ & 15 & 1,05 & $0,0255-0,03$ & $40-41$ \\
\hline 4)Internal carotid artery & $0,15-0,25$ & 75 & 1,05 & $0,0255-0,03$ & $40-41$ \\
\hline Arteriole & $0,005-0,01$ & 45 & 1,05 & $0,0255-0,03$ & $40-41$ \\
\hline $\begin{array}{c}\text { Veins: } \\
\text { 1) Cava }\end{array}$ & $0,5-3$ & $10-20$ & 1,05 & $0,0255-0,03$ & $40-41$ \\
\hline 2) Venule & $0,004-0,005$ & $0,3-1$ & 1,05 & $0,0255-0,03$ & $40-41$ \\
\hline Capillaries & $0,00045-0,0007$ & $0,2-0,3$ & 1,05 & $0,0255-0,03$ & $40-41$ \\
\hline
\end{tabular}

For the second problem, the blood flow in vein is modeled numerically, which gives an opportunity to make a picture of the flow cased by stenosis. It complements the information which might be received in experimental researches and allows determining the effect of a wall shape, flow type and character on the disease's degree. Such parameters as distribution of velocity along axis, vessels diameter, density, blood viscosity for the determination of physiological and pathological conditions in vessels. The special flow characteristic in vessels affected by stenosis is the appearance of intermediate regimes of flow, which are considered as pathological in contradistinction to normal flow regime. Due to growth of atherosclerotic plague the narrowing of canal is happening, which in its turn, leads to velocity rise in vessel and appearance of turbulence regime, which has bad effect on vessels of brain. In vessels of brain laminar flow appears in the low values of blood speed. In turn, flow with stenosis such features as division of flow on further eddy zones in vessels appears. The data for the numerical modeling is taken from the table 1 $[3,4,5]$.

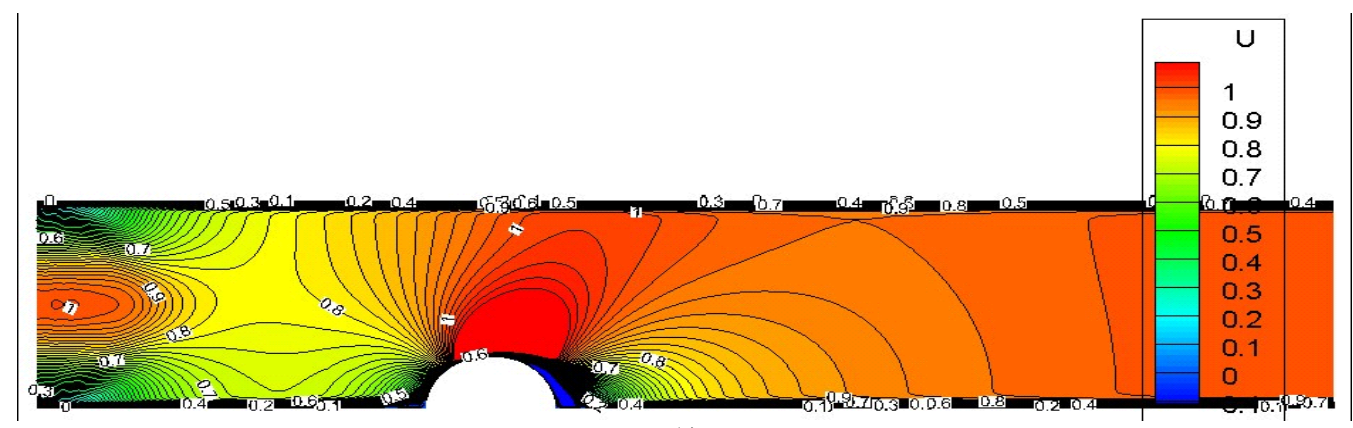

A)

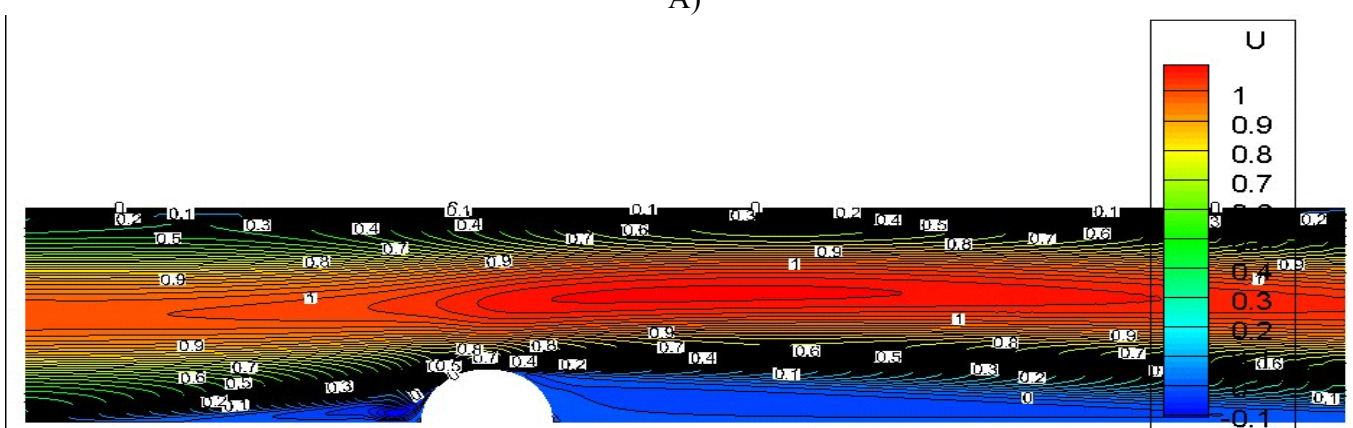

B)

Figure 6 - Contour of velocity field with stenosis for speed $v=15 \frac{\mathrm{cm}}{\mathrm{s}}$ in different time layers $\left.(\mathrm{A}) \mathrm{t}=0, \mathrm{~B}\right) \mathrm{t}=10$ ). 


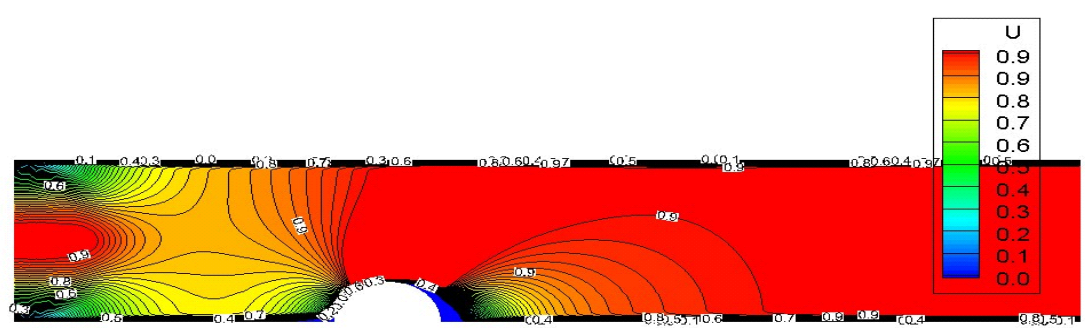

A)

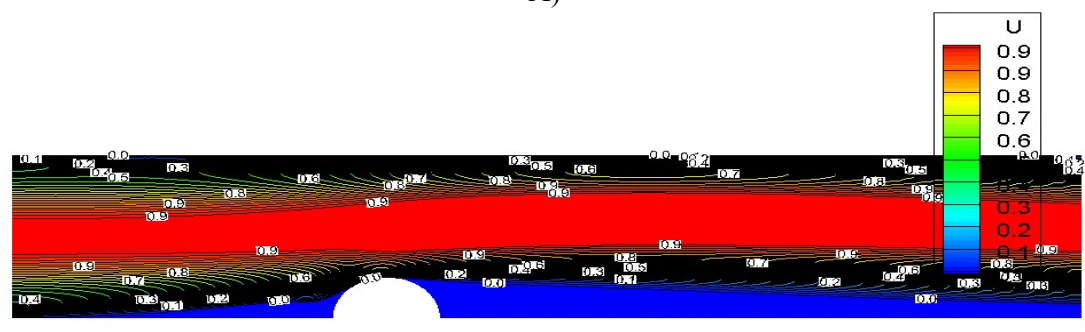

B)

Figure 7 - Contour of velocity field with stenosis for speed $v=20 \frac{\mathrm{cm}}{\mathrm{s}}$ in different time layers $\left.(\mathrm{A}) \mathrm{t}=0, \mathrm{~B}\right) \mathrm{t}=10$ )

The estimated area is given at figure 6, which shows an atherosclerotic plague. For the numerical modeling of stenosis in brain vessel the next parameters are used: vessel diameter $D=0.01 \mathrm{~mm}$., kinematic viscosity $\mu=0,03 \frac{\mathrm{gr}}{\mathrm{cm} \cdot \mathrm{s}}$.

At figures 6-8 the velocity field of blood flow with atherosclerotic plague in vessel of brain is presented for different time layers and different velocities. As it can be seen from the figures, vortex field appears behind the atherosclerotic plague. Wherein, blood velocity grows, making vortex field in zone of narrowing of vessel, which in its turn can transformed in turbulence flow. In the end, the atherosclerotic plaque can increase in size. At figures 9-11, presents the different vertical velocity profiles with stenosis for different numbers of velocity. At figures 10-11 are shown the velocity profiles before passing atherosclerotic plaque. The fracture in the velocity profile at figure 12 indicates the appearance of tearing off zone.

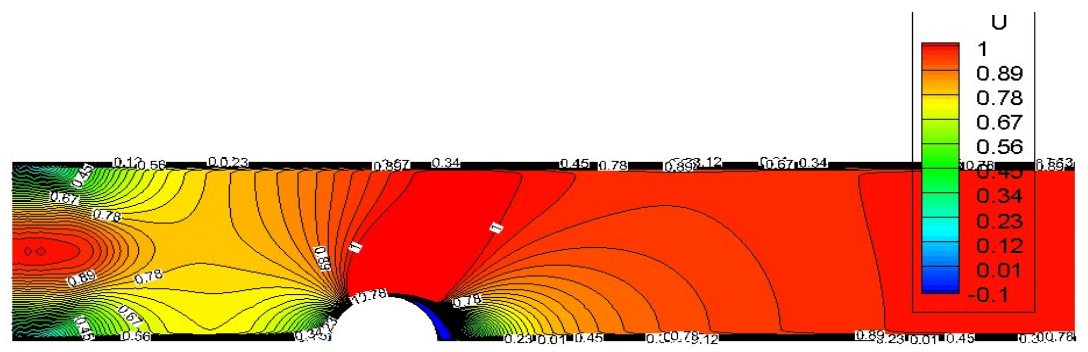

A)

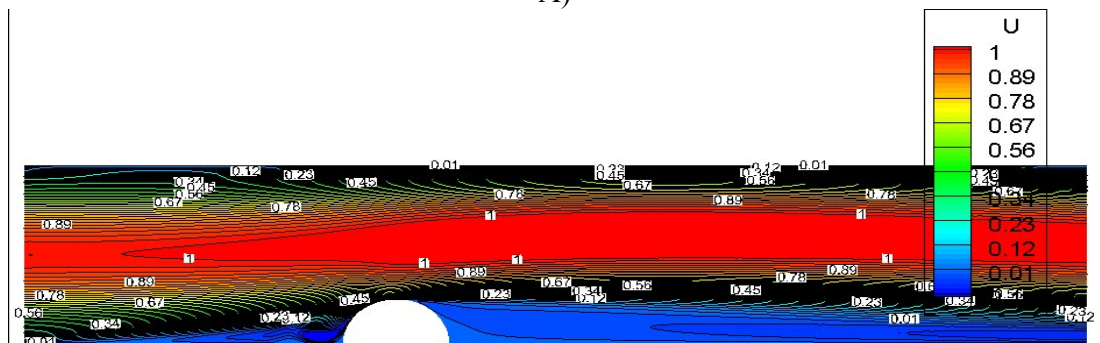

B)

Figure 8 - Contour of velocity field with stenosis for speed $v=25 \frac{\mathrm{cm}}{\mathrm{s}}$ in different time layers $\left.(\mathrm{A}) \mathrm{t}=0, \mathrm{~B}\right) \mathrm{t}=10$ ). 


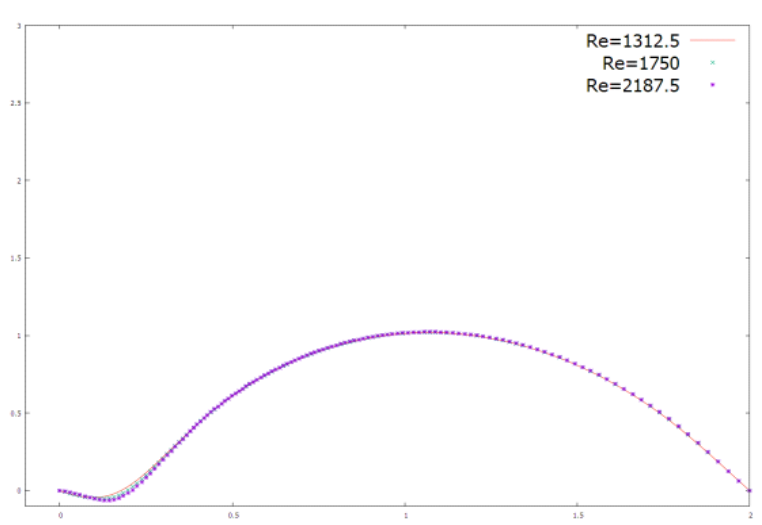

Figure 9 -Vertical velocity profile with stenosis for different velocity $\mathrm{x}=2,56$

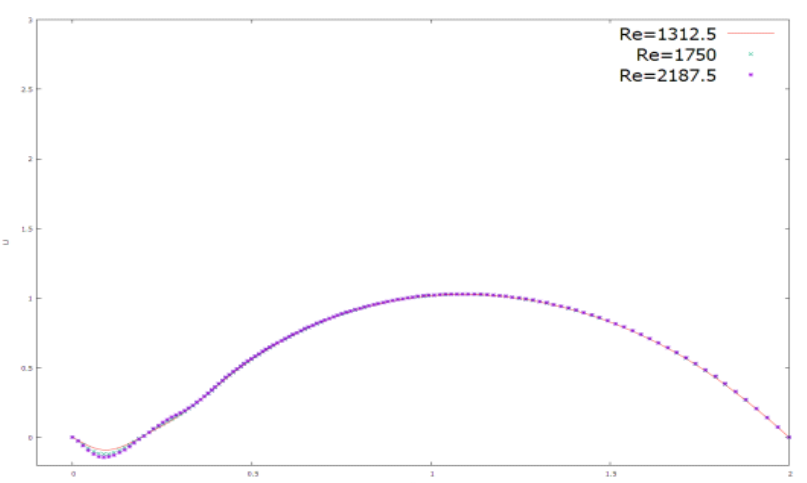

Figure 10 -Vertical velocity profile with stenosis for different velocity $\mathrm{x}=2,85$

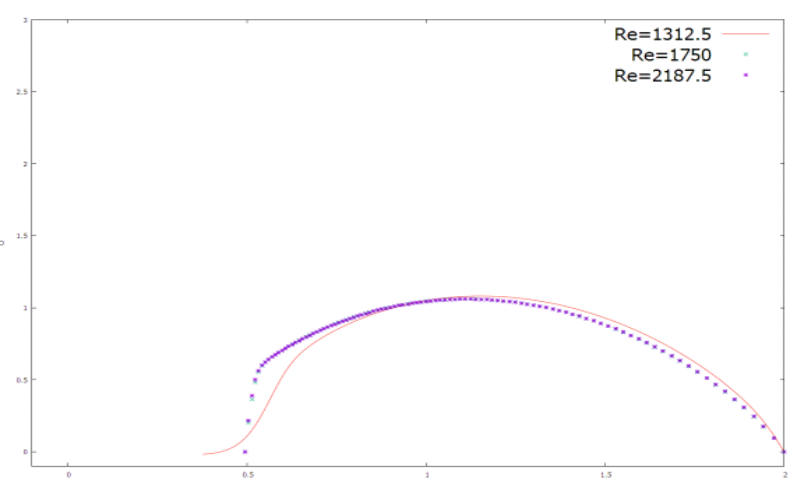

Figure11 -Vertical velocity profile with stenosis for different velocity $\mathrm{x}=3,45$

As plaque grow, the lumen decrease gradually, which can lead to its complete occlusion with time. The plagues growth is dangerous because its part can tear off and travel through bloodstream getting into a smaller vessel and plugging it up.In this type of stenosis, the violation of the blood supply in particular brain area happens. In slow stenosis development of arteries, the cerebrovascular insufficiency develops. The main manifestations are disturbance of memory, eyesight, speech, changes in emotional sphere, different movement disorders.

\section{Conclusion}

The numerical method is tested in example of Poiseuilleflow. According to numerical result the graph of Poiseuille flow profile, the numerical method gives satisfactory result. It allows us to apply this numerical method to the blood flow problem in vessels of brain with pathological diseases which we are interested in.

Numerical modeling of atherosclerotic plaque in vessels of brain gives an opportunity to make a picture of flow, which caused by stenosis.Further information can be obtained from experimental researches, which allows us to determine type, character of flow and degree of disease. For the detection of physiological and pathological processes in vessel, important parameters are velocity distribution along the axis and drop of pressure. One of the characteristics of blood flow in blood vessels affected with stenosis is the appearance of eddy flow regime, which pathology is comparing with a normal blood circulation. In laminar regime of blood flow in vessels of brain the flow occurs in low values of speed's numbers. When there is a stenosis in vessels a blood consumption decrease greatly because of eddy zones appearance. Taking the Navier - Stokes equation as a base in this work, movements of blood in vessels of brain are modeled numerically. The behavior of the blood flow is researched with different velocity, availability of atherosclerotic plaques that allows to diagnose disease at early stages and to choose right ways of therapeutic treatment and prevention. The results of this work might be used in solving of tasks of clinical medicine.

\section{References}

1. Skobtsov Yu.A., Rostov Yu.V., Overko B.S. Modelirovanie i vizualizatsia povedeniya potokov krovipri patologicheskikh protsessakh - Donetsk: Izatel'Zaslavskiy A. Yu. - 2008. - 212 p..

2. Zabielski L., Mestel A. Unsteady blood flow in a helically symmetric pipe // Journal of Fluid Mechanics. - 1998. - Vol. 370. - P. 321- 345. 
3. Smirnov V. M. Fiziologiyacheloveka. izd M.: Meditsina, 2002. -608 p.

4. Tsvibel' V., Pellerito Dzh. Ul'trazvukovoe issledovanie sosudov. izd. - M.: Vidar, 2008. $-644 \mathrm{p}$.

5. Tazyukov F. Kh., Dzhafar M. Khassan, Khalaf Kh. A., Snigerev B.A., Safa Kh. Abdul Rakhman Techeniekrovi $\mathrm{V}$ simmetrichnoi krovenosnoiarterii so stenozom // Rossiyskiy zhurnal biomekhaniki. - 2012. - Vol. 16. - No 1(55). - P. 46-56.

6. Schlichting G. Teotiya pogranich negosloya. izd. - M.: Nauka, 1974. - 708 p.

7. Issakhov A., Mathematical modeling of the discharged heat water effect on the aquatic environment from thermal power plant //
International Journal of Nonlinear Science and Numerical Simulation. - 2015. - Vol. 16, No 5. - P. 229-238.

8. Issakhov A., Mathematical modeling of the discharged heat water effect on the aquatic environment from thermal power plant under various operational capacities // Applied Mathematical Modelling. - 2016. - Vol. 40, No 2. P. 1082-1096

9. Issakhov A. Large eddy simulation of turbulent mixing by using 3D decomposition method // J. Phys.: Conf. Ser. - 2011. - Vol. 318. No 4. - P. 1282-1288.

10. Chorin A.J. Numerical solution of the Navier-Stokes equations // Math. Comp. - 1968. Vol. 22. - P. 745-762. 